\title{
MOBILE ELEMENTS AND DNA REPEATS
}

\author{
by
}

\author{
ROY J. BRITTEN
}

\author{
Department of Biology, California Institute of Technology \\ 101 Dahlia Avenue, Corona del Mar, California 92646, USA \\ Staff Member Carnegie Institution of Washington
}

Keywords: Transposons evolution vertebrate genome DNA-agar

\section{INTRODUCTION}

It now seems very likely that many repeated DNA sequences are mobile genetic elements (transposons) or were introduced into the genome through the action of transposons. This is very probably the case for interspersed repeats but less certain for tandem arrays which might have been generated by events of unequal crossing over. Thus it appears that mobile genetic elements have affected DNA sequence organisation in nearly every kilobase of the genome of most higher organisms. Recognition of the phenomena has been slow to come because of their complexity and their revolutionary implications for our understanding of genomic evolution. Our current insights depend on the work of MCCLINTOCK on corn genetics, on the modern studies of the genetics of mobile elements in bacteria, yeast and drosophila as well as the studies of genome organisation and repeated sequences in higher organisms.

This note is not a review and the intention is only to touch on aspects of the early repeated sequence studies which are scattered in inaccessible literature. The focus is on the parts of the earlier work that relate to current knowledge of movable genetic elements. As it turns out these measurements have significant implications for vertebrate genomes which need examination by modern methods. This note is also a brief summary of accomplishments in the years immediately following my visit to the Carlsberg
Laboratory as a guest of HEINZ HoLTER and is intended to convey appreciation for that year and suggest its influence and great value for me.

\section{BACKGROUND}

In 1961 the DNA-agar procedure was introduced $(1,2,3)$ and used for the measurement of hybridisation of DNA between the genomes of higher organisms. By 1963 a significant number of measurements had been made. An interesting example is shown on Figure 1. This figure is reproduced because it reflectsa significant aspect of the evolutionary history of mobile elements (discussed below). At the time of its publication the existence of repeated sequences was unknown.

The thermal stability of DNA/DNA hybrids is a measure of the extent of sequence match. The measurements of MARTIN and HOYER (25) and WALKER (33) showed that there was a large amount of sequence divergence between the members of families of repeats within one species and that the degree of divergence increased in interspecies comparisons as a function of the time since the last common ancestor existed. WALKER (33) had also shown that a specific subset of DNA fragments was responsible for the measured hybridisation.

Bolton, Hoyer and MCCARThy $(2,23)$ also showed that the same set of sequences hybridised regardless of the tissue from which 


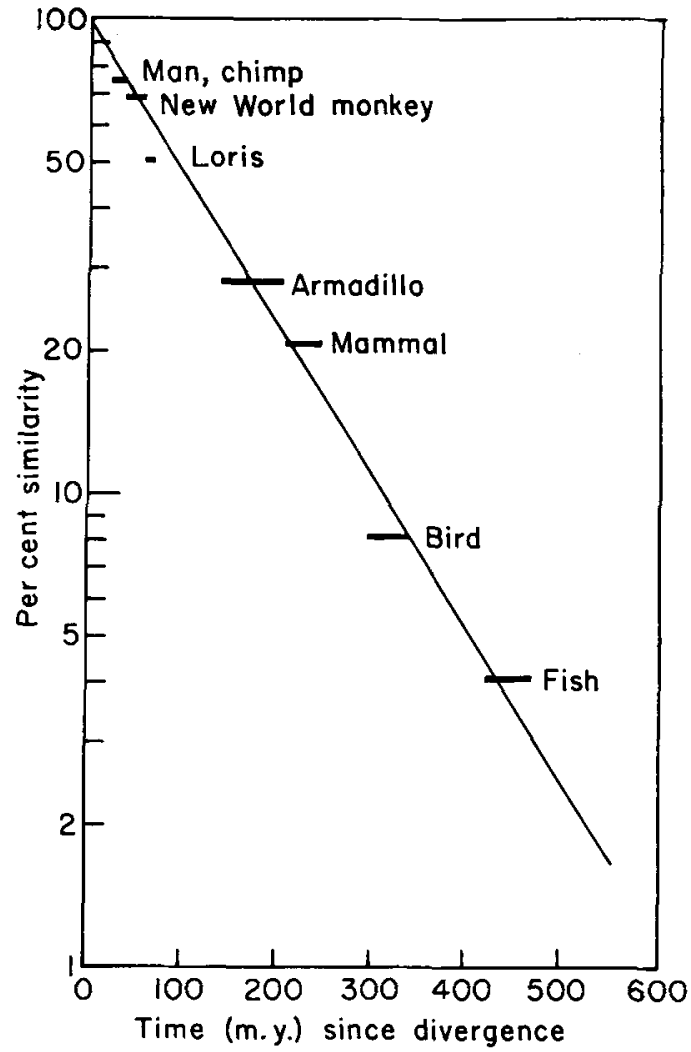

Figure 1. Estimation of the fraction of repeated sequences held in common between rhesus monkey DNA and the species listed.

This figure is reproduced from the original work of BOLTON, MCCARTHY and HOYER (1). The abscissa is total time since the last common ancestor with Rhesus monkey (both lines summed). The best dates that might now be used are (M. GoodMAN, personal communication): man, 50-80; new world monkey, 80-110; loris, 100-130; armadillo, 140-180; mammal (mouse), 140-180; bird (chicken), 560-640; fish (bony), 640-800. If used, these dates would increase the scatter of the data on the figure but would not affect the basic conclusion.

For this measurement Rhesus monkey DNA was immobilised by boiling, mixing with an equal volume of melted 10\% agar and sudden chilling. Labelled rhesus DNA fragments hybridised to about $25 \%$ extent with the bound rhesus DNA after incubation overnight at $60^{\circ} \mathrm{C}$ in $2 \times \mathrm{SSC}(0.3 \mathrm{~m} \mathrm{NaCl}, 0.03 \mathrm{M}$-sodium citrate). Bound DNA at $220 \mu \mathrm{g} / \mathrm{ml}$ and the Cot is about 32 , thus nearly completely reassociating repeats with 1,000 copies and probably half reassociating those with 100 copies.

Unlabelled fragments of the DNA of the various species was added in a fourfold excess over the bound rhesus DNA. The ordinate is the fraction of the labelled rhesus DNA that hybridised compared to the control without added unlabelled DNA. A decrease in "similarity" is best interpreted as a reduced number of members of repeated families (compared to the number of members in rhesus DNA) which are similar enough in sequence to hybridise with rhesus DNA and compete with the labelled rhesus fragments. The simple exponential decay of similarity with time since shared last common ancestors is presumably the result of a "single hit" process of loss or gain of repeated DNA sequences. the DNA was extracted, as expected for genomic DNA. In addition they showed that the sequences responsible for the hybridisation were transcribed in total cellular RNA and that RNA from different tissues showed partly overlapping and partly distinct hybridisation. This observation is entirely consistent with current work which shows that different families of repeated sequences are expressed to different extents in different tissues and cell types (32). We do not

Figure 2. The reassociation process for eukaryotic DNA as viewed in 1965 (12). The original caption follows:

Schematic diagram illustrating renaturation process seen with DNA from higher organisms. A. A short length of native DNA, with helix uncoiled for clarity. Heavy lines and letters identify sequences frequently repeated; only these sequences stand a reasonable chance of finding complementary partners in the renaturation process. Lengths shown for repeated regions andtheir spacing are symbolic. It is known that most sheared pieces (B) contain one such region and probably more. Structures in B represent denatured sheared DNA. A few of the possible fragments from the sample of DNA in A are shown. C. Result of renaturation of denatured high-molecularweight DNA. Very few possible complementary pairing regions find partners because of different distribution of pairing regions in various stretches of DNA. D. Renaturation of denatured sheared DNA. With only a few repeated sequences per sheared piece there is freedom for complementary partners to find each other. (Diagrams are not intended to indicate degree of precision of matching or length of re-formed complementary regions). 


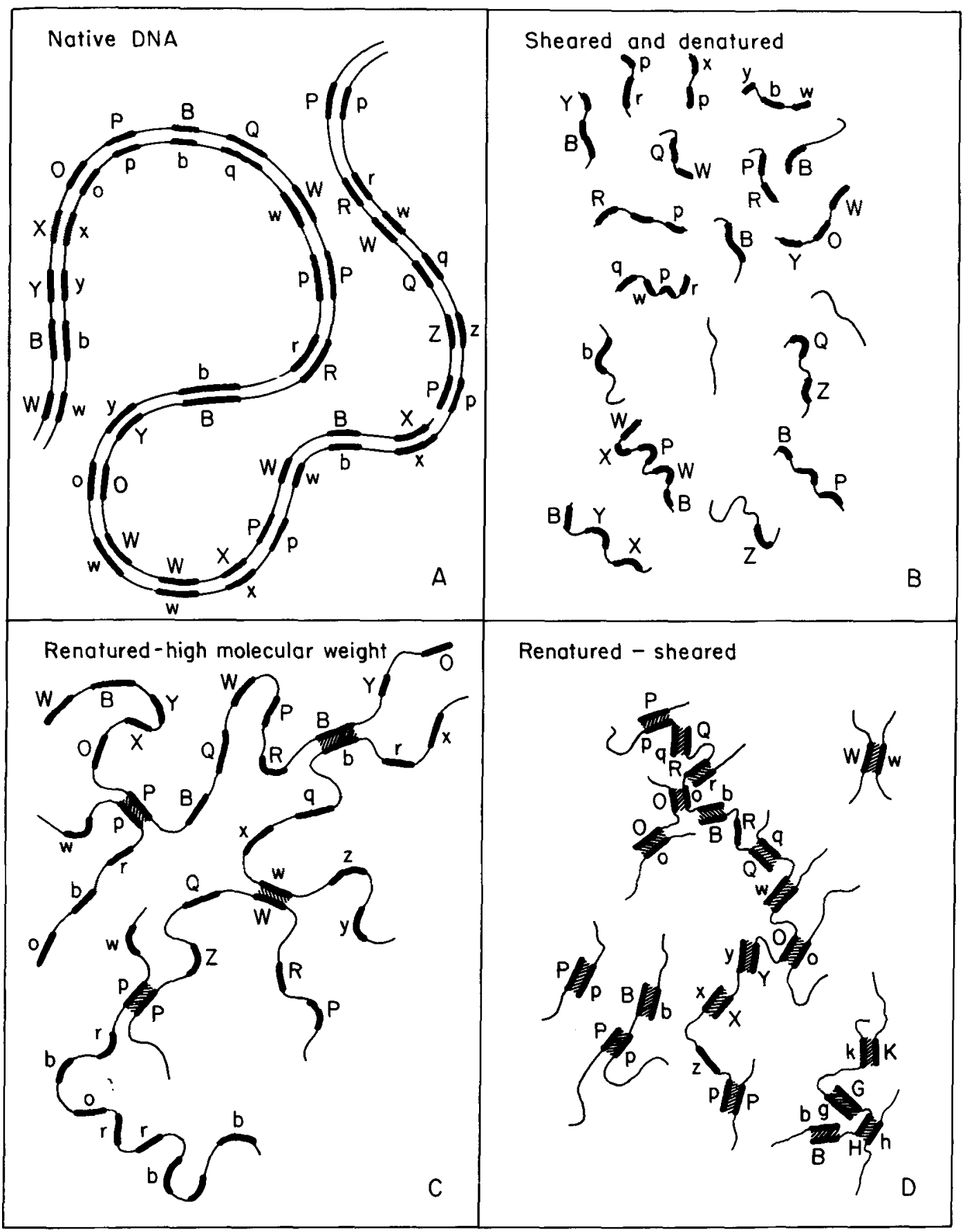


yet know the reason for the striking modulations of repeated sequence transcription in differentiation and development, but the implication is that some of the transcribed repeats have a significant role. It is now clear that many repeat transcripts appear in nuclear RNA or egg RNA and few reach polysomes $(13,14)$.

The DNA-agar measurements initiated the events leading to the proposal of the existence of repeated sequence families $(10,12)$. More important for the purposes of this note were the measurements of interspecies relationships which made possible the early recognition that the repeats were undergoing dynamic processes of evolutionary change.

In the spring of 1964 I decided to attack the question of why the DNA-agar hybridisation rates were a thousand fold faster than expected. The first tests (by following optical reassociation in slabs of agar) showed that agar did not have a catalytic effect on the process. Reassociation was also examined between labelled short (sheared) DNA fragments and large fragments using molecular sieve agar gel columns to separate the long and short fragments and thus to assay hybridisation. This procedure convincingly showed solution reassociation at expected rates and went further to establish that the rate calculations were correct. However, it did not work well with animal DNA due to large losses by filtration on the gel columns resulting from (as it turned out later) network formation in the DNA deriving from interspersed repeat reassociation (see Figure 2). Other methods superceded and these early measurements were never published. They were significant because they established that the rate of reassociation of animal DNA was intrinsic in the animal DNA itself and not an artifact.

In the summer of 1964 BILL HOYER (then at NIH) offered some mouse embryos as suitable material for the purification of long DNA. This choice of material was very fortunate since MIKE WARING then joined the work and together we performed a series of $\mathrm{CsCl}$ ultracentrifugation studies on this DNA. This work procedeed rapidly to the purification of the mouse satellite and the measurement of its rate of reassociation in solution by optical techniques (34). The rate of reassociation indicated a repeat length of about 300 nucleotides which has been fully confirmed.

Reported in this first paper (34) are solution hybridisation measurements between short fragments of labelled mouse DNA and networks which were subsequently trapped by simple filtration. This procedure does not work well with bacterial DNA since the interspersion of repeats was necessary in the formation of networks and DNA trapping.

In this period $\mathrm{CsCl}$ centrifugation measurements showed that calf DNA and garden pea DNA could be denatured and incubated together and nevertheless formed separate networks which yielded two distinct hypersharp bands. We concluded that network formation was DNA sequence dependent and due to reassociation of repeats rather than non-specific aggregation. More closely related DNAs such as those of calf and salmon which share a few repeated sequences do not form separate hypersharp bands when incubated together though they do when incubated separately, and subsequently mixed.

In 1965 MIKE WARING returned to England and DAVE KOHNE joined to start the hydroxyapatite studies of the general occurrence of repeats in eukaryotes. The work on sequence arrangement stopped but was taken up again after several years with quantitative measurements of interspersion of repeats in calf DNA (11) and later in sea urchin DNA $(4,7)$. Later DNA sequence arrangement patterns were examined in more detail $(17,19,21)$.

\section{INTERSPERSION, SALTATORY REPLICATIONS AND SPECULATION}

A few quotations from the early work are assembled here to indicate how much was understood at the time regarding the evolution of the families of repeats. The following excerpts summarize part of the evidence for interspersion known in 1965 (12):

1. "Renaturation of animal DNA occurs between strands in solution as well as when one partner is partially immobilized by embedding in agar.

2. The resulting complementary strand pairs are only partly helical in structure, and the 
Original gene

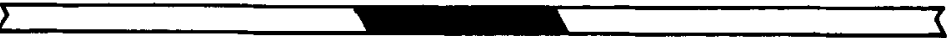

Mutation
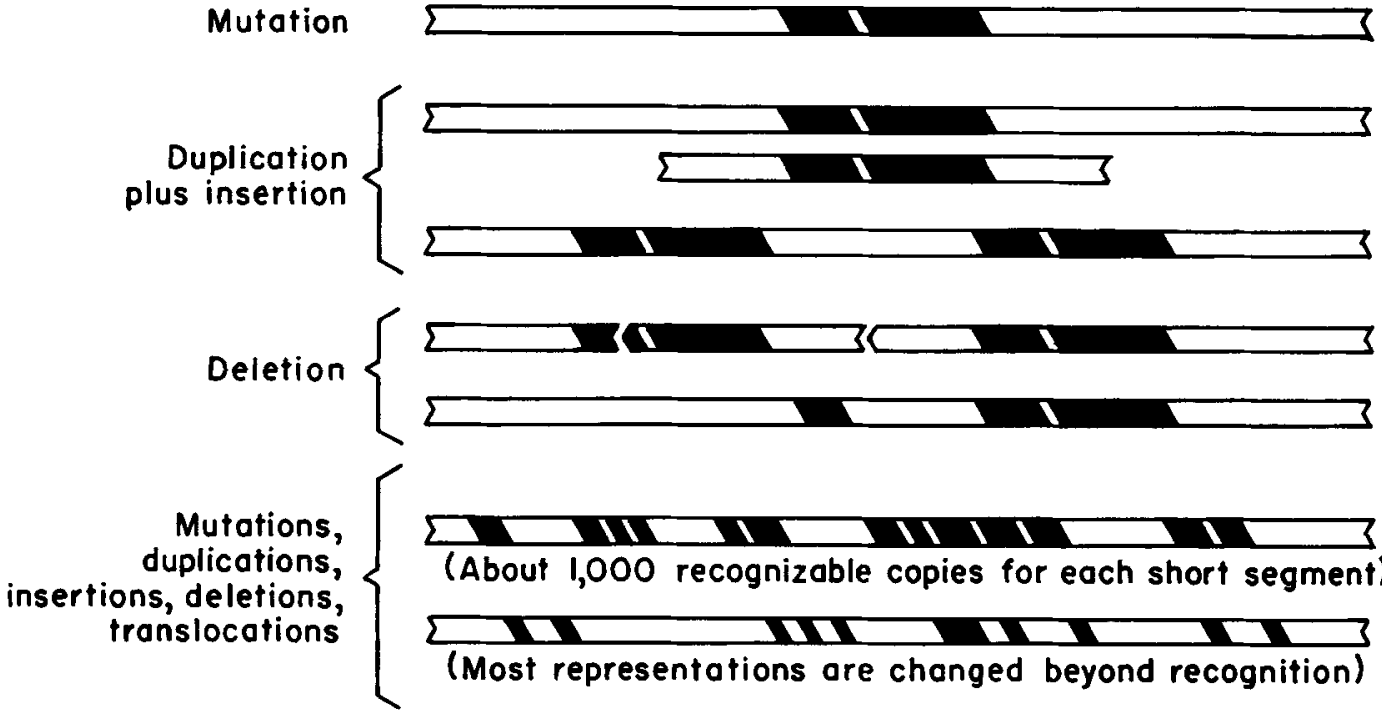

Figure 3. Suggested time course of repeated sequence evolution from 1965 (12). This model focussed on steps of duplication since the concept of saltatory replication had not yet been proposed. It was clearly understood that the products of the duplication were inserted throughout the genome. The elements being duplicated were thought to be "genes" since it was not yet realized that a majority of the genome was ancillary to genes or independent of them. The original time estimates have been removed since they were not very good guesses. The black marking indicates the retention of recognizable sequence homology.

helical regions are disrupted at a lower temperature than that required to disrupt the helical structure of native DNA. ...

4. Renaturation of animal DNA in solution leads to the formation of extended structures or three-dimensional networks in which any given strand forms helical base-paired regions with a number of other strands.

5. The formation of such networks involves species-dependent specific pairing of complementary base sequences. ...

7. Even when the DNA of an animal cell is sheared into millions of pieces and denatured, a majority of the single-stranded fragments find complementary strands with which to renature in a relatively short incubation period."

Figures 2 and 3 represent the initial interpretation of the pattern of interspersion and how the pattern evolved. Only quantitative criticism can now be made of these diagrams. The single copy stretches in Figure 2 are too short relative to the repeats. The comment about 1,000 copies on Figure 3 actually represents a range from a few up to a million member sequences per family. One could also cavil with the use of the word gene on Figure 3.

The following comments were made on the evolution of repeats in 1964 (12).

"Since many DNA sequences exist in repetitive families composed of many members, new repetitions must be continually created during the evolution of a species. This becomes evident when it is realized that each of two species that have diverged significantly maintains large amounts of internal homology or repetition, but has lost some interspecies homology. Thus, in at least one of the species (and presumably both) new sets of multiple repeated sequences have arisen. ...

Thus, the pattern of repetition in the genome of a particular animal would result from a balance of processes such as duplication, translocation, point mutation, and deletion of nucleotide sequences, as suggested in Figure 3. 
From this view it is useful to regard the repetitive DNA sequences as relatives of each other-more or less distant, depending on their particular history and the selection pressure against or favoring change. Families of descendants could exist with any number of members from one or two to tens of thousands. Whether the relationships are created gradually by fairly common single duplications or catastrophically by rare events of extreme multiplication remains for the future to tell. ...

The probable relation between gene duplication and the rate of evolution is also of interest. Once a gene has been duplicated, the risk of deleterious mutation is decreased. Further, if we may consider one copy (the effective gene) as being conserved relatively unchanged as a result of selection pressure, so that the function it specifies is neither lost nor adversely modified, other copies would be free to mutate as long as deleterious gene products did not result. These copies would initially have the capacity to specify a complete gene product - for example, an enzyme or part of a physiological system. As mutations occurred in the copies some elements might be lost or modified and other elements maintained. The capacity of some copies to specify the original function might well disappear entirely in the course of time in lines of animals that would not, on this ground, suffer a selective disadvantage.

The development of a gene specifying a new function from remaining elements of an older gene that had been protected from adverse selection pressure by copying seems to be far more probable than its development de novo from a random nucleotide sequence, and would represent an evolutionary mechanism of consequence. A great source of variety in new structures might result from the combination (by translation of parts of duplicated genes) of elements from several pre-existing genes."

The annual report published in 1966 (8) covers the formal exploration of repeated sequences (10) and is the origin of the term saltatory replication (8):

"Saltatory replication. The paucity of DNA with a small frequency of repetition has definite implications for the mechanism of production of repeated sequences. Why is it that a few sequences are copied many times, but the great majority of sequences have been copied little, if at all?It appears that particular sequences must be favored in the duplication process."

In the following year, 1967, more evidence for saltatory replication was collected and the following comments were made (9):

"Suppose that an event occurs in which a large number of unexpressed genes, or better, a class of genetic potentiality, is produced in the genome but only a small part appears phenotypically. The small, expressed part, "the top of the iceberg," if favorably selected will introduce the as yet unexpressed class into the population. In effect a hidden package of potential genetic effects would have been selected which after spreading through the population could lead to radically new features that could not result from a detailed balance of mutation and selection.

A saltatory replication producing 100,000 copies of the right sort of gene is a candidate for a genetic event with immense potentiality. Not all the copies would be initially expressed. Perhaps not many ever would be. But mutation, translocation, and recombination with other genes would yield a whole range of potential genetic activity which would perhaps turn up long after the appearance of the first effects that resulted from the saltation. If the early effects were selectively advantageous the whole set of products of the saltatory replication would be introduced into the population. The dynamics of selection would be fundamentally altered. The species might then appear to take a surprising and even an apparently purposeful course of evolutionary change. Owing to the great multiplicity of copies, their selctive elimination might be impossible short of eradicating the species. Potentialities important in the longer term would then have an opportunity to survive and reach fruitful expression.

Events of this general type would be capable of causing a striking divergence of two genetically isolated populations even though the environments in which the two populations existed were identical.

The wide occurrence of families of repeated sequences and the implication of saltatory events in their production as well as in evolutionary processes indicates that sudden events may 
be far more important to evolution than we have heretofore suspected."

This quotation from 1968 (10) prefigures the use of the Drosophila $P$ factor for transformation (31).

"Even if saltatory replications are ... rare, ... certain stages of the process may be relatively common. ... the following steps seem necessary. (i) A sequence undergoes manyfold replication; (ii) the copies are integrated into the chromosome; (iii) they become associated with a favorable genetic element, and (iv) they are disseminated through the species by natural selection.

Each of the succeeding stages is likely to have a very low probability of occurrence, and thus the actual event of manyfold replication may occur fairly commonly and, in principle, be observable in individual organisms. ... It does not seem impossible that, some time in the future, saltations may be artificially introduced into populations as mutations can already be."

Further speculation on the possible evolutionary role of repeats came in 1971 (6).

"The gene regulation model proposed earlier (5) provides a possible molecular level interpretation of the programming of gene activity in development (16). It indicates how specification of particular cell lines in development could occur in the processes of localization and induction. A basic property is the potentiality for evolutionary modification of the regulatory systems that were envisioned. In this model important parts of the regulative relationships depend on the location of sequences in the genome, and a variety of changes can be caused by sequence translocation. As a result, a great many individual cellular properties could be affected by genomic alterations of a type which may occur relatively frequently, namely, translocations and chromosomal rearrangements. It is quite plausible that selectively favorable changes of the former type are much more frequent. Furthermore, the model regulatory system is receptive to the incorporation of new sequences and sets of sequences, and through these additions can achieve new functional interrelationships. It can grow as well as become rearranged. Whether or not the particular gene regulation system that we have proposed turns out to occur in living systems, the properties of growth and change by genomic rearrangement are likely to be necessary attributes of evolving regulatory systems."

\section{EVOLUTIONARY FREQUENCY CHANGE AND THE RELATIONSHIP BETWEEN REPEATS AND MOBILE ELEMENTS}

The remainder of this note focusses on the implications of the data of Figure 1 and a few more recent observations that contribute to its interpretation.

The observation that repeated sequences are both interspersed and change in frequency over evolutionary time implies a process of insertion (and deletion) of relatively short sequences in many locations in the genome. This is formally equivalent to duplication and transposition but we know almost nothing about the mechanism of these processes in eukaryotes. However a few mobile elements have been identified in yeast (29) and Drosophila (30), and a smaller number among vertebrates and sea urchins $(18,20,22)$.

It is known that the positions of many repeats are unstable in the Drosophila genome. When the DNA of two strains is compared any given repeat in one strain is likely to be absent from the same location in a second strain (35). However the evolutionary frequency changes which might result from this frequent transposition are not known. There probably exist a number of different processes. Several classes of transposable elements are known and one example "levitates" segments of DNA (which are not strictly a part of the transposon) from one location to another. Obviously we know much less about the mechanisms and the movable elements in other eukaryotes.

We know that the processes of repeated sequence insertion, transposition and evolution result in a quite different DNA sequence organisation for typical eukaryotes as compared to Drosophila. Drosophila and some other insects (not even all Diptera) have a long period pattern of interspersion where the repeats are typically several kilobases in length $(15,24)$ while most eukaryotes that have been examined show a short period pattern in which the repeats are typically a few hundred nucleotide pairs in length (17). Thus there is reason to believe that 
the mechanisms involved and the characteristics of transposons (particularly length) are also quite different in most eukaryotes.

In our present state of ignorance any information on the evolution or properties of eukaryotic repeats in general is valuable for the purposes of orientation and planning further studies. Figure 1 is valuable because of the striking nature of the relationship: a simple exponential decay of repeat sequence interspecies hybridisation with evolutionary time. This observation has been subsequently repeated by the same technique using different sets of species $(9,25)$. When the exponential decay was first observed (1) it was suggested that DNA sequence loss might be the explanation. It now appears that that comment was correct but what is lost in a short time or "event" is not asingle bit of DNA sequence but most of the members of shared families of repeats.

A simple quantitative argument leads to this conclusion. Consider the number of events required for loss of hybridisation between a family of repeats in one genome and the same family in the genome of a related species. If the divergence is due to single events of base substitution many events are required per repeat sequence. Perhaps 50 mismatches in a 300 nucleotide long repeat would lead to failure of hybridisation under the conditions used. Of course the loss of recognition between many family members is required and the total number of events is very large, possibly 50,000 base substitutions per family.

A process in which a large number of events is required to create an observable effect is called a multiple hit process and the effect shows as a large lag time before any change occurs followed by an ever steepening rate. This is quite the opposite of an exponential decay which has its steepest slope at the beginning. The effect is quite sensitive and has been examined in detail in radiation damage studies. If only two events are required for loss an exponential plot of the survivors vs exposure shows a lag followed by an increasing rate which finally approaches a straight line. This line extrapolates to twice the starting population at time zero. The multiplicity of the process can be accurately determined by this extrapolation method. If the straight line projects to the starting value ( $100 \%$ at time zero) it is shown to be a single hit process.

Obviously the application of this argument to Figure 1 shows that repeated sequence sharing between related species has been lost by a single hit process. What is a hit? It is not the loss of a family member in one species or addition in another because this would be a multiple hit process with a multiplicity equal to the number of members of a typical repeat sequence family. Computer modelling (BRITTEN, unpublished) has confirmed these arguments either for base substitutions in already divergent families or for events of loss or addition of single family members at a moderate rate.

The only explanation we have been able to develop for the data of Figure 1 is that the hits are the loss (and gain) of whole families of repeats in relatively short evolutionary times. This revolutionary kind of "hit" is apparently the dominant process of evolutionary divergence of repeated sequence relationships among vertebrate species including higher primates. It seems very likely to be due to the insertion of many copies of some sort of mobile genetic element (and loss of other sets).

Very few examples of such potential mobile elements have yet been observed among vertebrate genomes but it is likely that many will be discovered as soon as they are directly searched for. Examples of repeated sequence families undergoing this process have been directly observed among sea urchins $(26,2728)$ where individual families of repeats have been studied with cloned probes. In several cases frequency changes of a factor of ten to twenty have occurred in something like 20 million years. This is a sufficiently rapid rate to be effective as a "hit" on the long evolutionary time scale of Figure 1.

In many of the cases examined there are 50 or 100 members of the family (not 1 or zero) in the species with the small number of family members. A few hundred or thousand members are typically observed in the other species with the large number of family members. It is clear that a small subset of all sequences are multiplied and inserted at remarkably high rates in most animal genomes. 


\section{REFERENCES}

1. Bolton, E.T., R.J. Britten, T.J. Byers, D.B. COWIE, B. HoYer, Y. Kato, B.J. MCCARThy, M. MIRANDA \& R.B. Rober TS: Biophysics. In: Carnegie Institution of Washington Yearbook No. 63, 366-397 (1964)

2. Bolton, E.T., R.J. Britten, T.J. Byers, D.B. COWIE, B. HOYER, B.J. MCCARTHY, K. MCQUILLEN \& R.B. ROBERTS: Biophysics. In: Carnegie Institution of Washington Yearbook No. 62, 303330 (1963)

3. BOLTON, E.T., R.J. BRITTEN, D.B. COWIE, B.J. MCCARTHY, J.E. MIDGLEY \& R.B. RobertS: Biophysics. In: Carnegie Institution of Washington Yearbook No. 61, 244-293 (1962)

4. BRITTEN, R.J.: DNA sequence interspersion and a speculation about evolution. In: Evolution of Genetic Systems. H.H. Smith ed., Gordon and Breach, New York 80-94 (1972)

5. BRITTEN, R.J. \& E.H. DAvidSON: Gene regulation for higher cells: a theory. Science $165,349-358$ (1969)

6. BRITTEN, R.J. \& E.H. DAvidSON: Repetitive and nonrepetitive DNA sequences and a speculation on the origins of evolutionary novelty. Quart. Rev. Biol. 46, 111-138 (1971)

7. BRITTEN. R.J. \& E.H. DAvidSON: Recent studies on moderately repetitive DNA sequences. In: Molecular Genetics and Developmental Biology. M. Sussman ed., Prentice-Hall, New Jersey 5-27 (1972)

8. BRITTEN. R.J.\& D.E. KoHNE: Nucleotide sequence repetition in DNA. In: Carnegie Institution of Washington Yearbook No. 65, 78-87 (1966)

9. BRITTEN, R.J.\& D.E. KoHNE: Repeated nucleotide sequences. In: Carnegie Institution of Washington Yearbook No. 66, 73-88 (1967)

10. BRITTEN, R.J. \& D.E. KoHNE: Repeated sequence in DNA. Science 161, 529-540 (1968)

11. BritTen, R.J. \& J. SMith: A bovine genome. Carnegie Institution of Washington Yearbook No. 69, 378-388 (1970)

12. BRITTEN, R.J.\&M.J.WARING: Renaturation of the DNA of higher organisms. In: Carnegie Institution of Washington Yearbook No. 64, 316-333 (1965)

13. Costantini, F.D.. R.J. BRItTen \& E.H. DavidSON: Message sequences and short repetitive sequences are interspersed in sea urchin egg poly (A) RNAs. Nature 287, 111-117 (1980)

14. Costantini. F.D., R.H. Scheller, R.J. Britten \& E.H.DAVIDSON: Repetitive sequence transcripts in the mature sea urchin oocyte. Cell 15, 173-187 (1978)

15. Crain. W.R., F.C. Eden. W.R. Pearson, E.H.
DAVIDSON \& R.J. BRITTEN: Absence of short period interspersion of repetitive and non-repetitive sequences in the DNA of Drosophila melanogaster. Chromosoma 56, 309-326 (1976)

16. Davidson, E.H. \& R.J. BritTen: Note on the control of gene expression during development. J. Theoret. Biol. 32, 123-130 (1971)

17. Davidson, E.H., B.R. Hough, C.S. AMENSON \& R.J. BRITTEN: General interspersion of repetitive with non-repetitive sequence elements in the DNA of Xenopus. J. Mol. Biol. 77, 1-23 (1973)

18. Deininger, P.L., D.J. Jolly, C.M. Rubin, T. FRIEDMANN \& C.W. SCHMID: Base sequence studies of 300 nucleotide renatured repeated human DNA clones. J. Mol. Biol. 151, 17-33 (1981)

19. GoldberG, R.B.. W.R. Crain, J.V. Ruderman. G.P. MoORe, T.R. BARNetT, R.C. Higgins, R.A. Gelfand. G.A. Galau. R.J. Britten \& E.H. DAvidson: DNA sequence organization in the genomes of five marine invertebrates. Chromosoma 51, 225-251 (1975)

20. Grimaldi. G. \& M.F. Singer: Members of the $\mathrm{Kpn} 1$ family of long interspersed repeated sequences join and interrupt a-satellite in monkey genome. Nucleic Acids Research 11, 321-338 (1983)

21. Graham, D.E., B.R. Neufeld, E.H. Davidson \& R.J. BRITTEN: Interspersion of repetitive and nonrepetitive DNA sequences in the sea urchin genome. Cell 1, 127-137 (1974)

22. LiebermanN. D. B. Hoffman-LiebermanN, J Weinthal. G. Childos. R. Maxson. A. Mauron. S.N. COHEN \& L. KEDES: An unusual transposon with long terminal inverted repeats in the sea urchin Strongylocentrotus purpuratus. Nature 306, 342-347 (1983)

23. MCCARTHY, B.J.\&E.T. BOLTON: An approach to the measurement of genetic relatedness among organisms. Proc. Natl. Acad. Sci. USA 50, 156-164 (1963)

24. Manning. J.E. C.W. Schmid \& N. Davidson: Interspersion of repetitive and nonrepetitive DNA sequences in the Drosophila melanogaster genome. Cell 4, 141-155 (1975)

25. MARTIN. M. \& B. HOYER: Thermal stabilities and species specificities reannealed animal deoxyribonucleic acids. Biochemistry 5, 2706-2713 (1966)

26. Moore. G.P. F.D. Costantini. J.W. Posakony. E.H. DAvidson \& R.J. BRITTEN: Evolutionary conservation of repetitive sequence expression in sea urchin egg RNAs. Science 208, 1046-1048 (1980)

27. Moore, G.P.. W.R. Pearson, E.H. Davidson \& R.J. BRITTEN: Long and short repeats of sea urchin 
DNA and their evolution. Chromosoma 84, 19-32 (1981)

28. Moore, G.P.. R.H. SCheller, E.H. Davidson \& R.J. BRITTEN: Evolutionary change in the repetition frequency of sea urchin DNA sequences. Cell $15,649-660$ (1978)

29. ROEDER.G.S. \& G.R. FINK: Transposable elements in yeast. In: Mobile Genetic Elements. J.A. Shapiro ed., Academic Press, New York (1983)

30. RuBIN, G.M.: Dispersed repetitive DNAs in Drosophila. In: Mobile Genetic Elements. J.A. Shapiro ed., Academic Press, New York (1983)

31. Rubin.G.M.\& A.C. SPRAdling: Genetic transformation of Drosophila with transposable element vectors. Science 218, 348-353 (1982)
32. Scheller, R.H., F.D. Costantini. M.R. KozLOWSKI, R.J. BRITTEN \& E.H. DAVIDSON: Specific representation of cloned repetitive DNA sequences in sea urchin RNAs. Cell 15, 189-203 (1978)

33. WALKER. P.M.B. \& A. MCLAREN: Specific duplex formation in vitro of mammalian DNA. J. Mol. Biol. 12, 394-409 (1965)

34. WARING, M. \& R.J. BRITTEN: Nucleotide sequence repetition: a rapidly reassociating fraction of mouse DNA. Science 154, $791-794$ (1966)

35. Young. M.: Middle repetitive DNA: A fluid component of the Drosophila genome. Proc. Natl. Acad. Sci. USA 76, 6274-6278 (1979) 